\title{
THE SEQUEL OF A CASE
}

(PUBLISHED IN VOLUME XLI OF THE TRANSACTIONS OF THE ROYAL MEDICAL AND CHIRURGICAL SOCIETY)

or

\section{IT H 0 T R I T Y,}

IN WHICH A COMMUNICATION EXISTED BETWEEN THE BLADDER AND INTESTINE.

BY.

CHARLES HAWKINS, F.R.C.S.,

CONSULTING SURGEON TO QUEEN CHARLOTTE'S HOSPITAL; INSPECTOR OF ANATOMY, ETC.

Received June 27th.-Read June 28th, 1859.

In the last volume of the 'Transactions' of the Society there is an account of a case, in which a communication existed between the bladder and intestine, where a calculus had formed in the bladder, which I removed by lithotrity.

The patient having since died, I have thought that the following account of what was found at a post-mortem examination, might prove of sufficient interest to occupy the attention of the Society for a short time.

On February 2d, 1858, the patient was reported to be quite free from calculus in the bladder; and there were no symptoms of stone from this time to that of his death, which took place on April 19th, 1859.

He continued to pass fæcal matter occasionally with his 
urine, and until within a few weeks of his death he daily washed out his bladder with warm water, by means of a syringe and catheter. About three or four months previous to his death his general health gave way; his digestive organs became impaired, accompanied with considerable irritability, and some mental disturbance; the symptoms exhibited previous to his death did not appear to depend upon the disease of the bladder or intestine.

Mr. Shield, of Hungerford, under whose care he was latterly, examined the body after death, and was good enough to send me an account of what he found, and also the bladder, and the portion of the intestine implicated in the disease, which are now in St. George's Hospital Museum. The following are the appearances they presented.

There was an opening in the bladder at the lower part of the posterior wall, of the diameter of a goose-quill, evidently not of recent date; the bladder corresponding to this aperture was intimately united by old adhesions to that part of the circumference of the sigmoid flexure of the colon that lies nearest to it. The aperture in the bladder communicated with the sigmoid flexure opposite their point of union. Above the point of communication of these two viscera, for the extent of about an inch, the canal of the sigmoid flexure was somewhat constricted; but this constriction was apparently due to the adhesion and subsequent contraction of these viscera, as beyond the point where the adhesion between them existed, the calibre of the sigmoid flexure appeared normal. Below the communication between the bladder and colon, the canal of the intestine was greatly constricted, to the extent of an inch and a half in length, admitting a tube through it of the size of the little finger. This stricture appeared to depend upon great condensation and subsequent cicatrization of the submucous and muscular tissues at that point. The mucous membrane of the intestine, above the seat of stricture, presented in many places pouches, varying in size from that of a pea to that of a filbert, and formed by protrusion of this coat externally. Opposite to the stricture it appeared 
to be healthy', but very densely convoluted. The rectum was very much dilated, and had, during life, evidently acted as a second bladder, as from the symptoms described by the patient, the urine used to accumulate there, and was discharged in considerable quantities per anum. The bladder was healthy, and did not contain any calculous matter. The kidneys were somewhat congested, but otherwise were in a normal condition.

The appearances described bear out the opinion I gave in my former paper.

The history of this case is a good example of the great value of lithotrity in the treatment of calculus in the bladder in complicated cases. I was enabled, by this operation, entirely to remove the stone, and relieve the patient of great suffering; and I think, considering the state of parts revealed by the post-mortem examination, that, had lithotomy been resorted to, it would not have been followed by the same amount of success.

Mr. Sydney Jones, in December last, exhibited at the Pathological Society a specimen very similar to that I have described, and where a calculus was formed in the bladder, with fæcal matter as a nucleus; but no attempts appear to have been made to remove it, extravasation being the immediate cause of death. Mr. Jones observes- "The cause of death was the existence of calculus in the bladder. Had not this impediment to the escape of urine from the bladder been caused by the presence of this calculus, it is probable that the case would not have had so speedy a termination." 